\title{
Preoperative detection of ovarian cancer by color doppler ultrasonography and CA 125
}

\author{
Fawzia Hossain ${ }^{1}$, Md Nazmul Karim², Shah Md Mahfuzur Rahman², Nasreen Khan ${ }^{4}$, \\ Maruf Siddiqui ${ }^{4}$, Raihan Hussain ${ }^{5}$ \\ ${ }^{1}$ Bangabandhu Sheikh Mujib Medical University, Dhaka ${ }^{2}$ National Institute of Traumatology and \\ Orthopaedic Rehabilatation, Dhaka ${ }^{3}$ Institute of Public Health, Dhaka. ${ }^{4}$ DGHS, Dhaka. \\ ${ }^{5}$ Institute of Nuclear Medicine, Dhaka
}

\begin{abstract}
Purpose: Early detection of ovarian malignancy is of great clinical importance. The high mortality rate is due to the difficulties with the early detection of ovarian cancer. Current research attempted to assess the accuracy of Color Doppler Sonography and serum CA-125 level as diagnostic tool of ovarian tumor. Materials and Methods: In this cross-sectional study, 60 consecutive patients with ovarian tumor attending the Department of Obstetrics and Gynecology of BSMMU were recruited. Of the study participants $23.3 \%$ belong to $16-25$ year age group, 20\% belong to 26-35 years age group and $23.30 \%$ each were of 46-55 years and > 55 years age group. All the patients recruited were from in-patient department and had undergone surgery. Following excision, routine histopathology revealed $43.30 \%$ malignant $(n=26)$ and $56.7 \%(n=34)$ benign ovarian lesion. Data were collected from the clinical history form and bimanual pelvic examination, serum CA 125 levels, estimation of Resistance index (RI), Pulsatility Index (PI), Novel Index by CDS and post-operative histo-pathological findings were then recorded. Sensitivity, specificity, accuracy, positive and negative predictive value of the diagnosis made by CDS, CA125, in the discrimination of the benign and malignant ovarian tumors was calculated. Using Receiver operative characteristics analysis the accuracy of RI, PI, CA 125 and Novel Index in the diagnosis of ovarian tumor (benign or malignant) were assessed. Results: With the Cut-off of <.5, Resistance Index is found to be capable of detecting $92 \%$ of malignant cases (sensitivity 91.7), and could detect $89 \%$ (specificity 88.9) of benign cases correctly which translates in to $90 \%$ accuracy in the diagnosis of ovarian tumor. Predictive values for positive (84.6) and negative (94.1) tests were also found to be quite high. Pulsatility index was found to be moderate accuracy (63.3\%) with cutoff $<1$ for malignancy, however low predictive value for a positive test (38.5) questions its use. Both CA-125 and Novel Index showed similar level of sensitivity and specificity. Although Novel Index is derivative of CA125, Novel Index demonstrated better diagnostic accuracy and negative predictive value. The cutoff for CA 125 was mandated as 83.58. With the value the sensitivity is $76.9 \%$ and the specificity is $94.1 \%$. RI is found to be more sensitive in detection of positive cases (Malignant) and CA125 is found to be more accurate in detection of negative cases (Benign). However a combination could be tried to make a better detection. Conclusion: Color Doppler ultra-sonography and CA125 excels in different tasks, the study concludes in favor of concurrent use of the methods for improving efficacy and thus early detection of ovarian malignancy.
\end{abstract}

\section{Introduction}

In most of the cases ovarian tumor are diagnosed at an advanced stage, as insidious onset and progression of this tumor makes early diagnosis difficult. Early detection of ovarian malignancy is of great clinical importance. Ovarian cancer is characterized by few early symptoms, presentation at an advanced stage, and poor survival. It is three times more lethal than breast cancer ${ }^{1}$.

The high mortality rate is due to the difficulties with the early detection of ovarian cancer. Around
$80 \%$ of patients is diagnosed with advanced staged disease. In patients who are diagnosed with early disease (stage I or II), the 5-year survival ranges from $60 \%$ to $90 \%$, depending on the degree of tumor differentiation ${ }^{2}$.

Ovarian cancer if diagnosed pre-operatively as benign or malignant, it can be of great help for the gynecologist in planning the mode of treatment.

Detection of ovarian cancer at an early stage, when they are localized to the ovary, more than $90 \%$ of theses patient would have a survival longer than 5 
years after removal of tumor ${ }^{3}$. Research in animal model also signifies the role of early detection on prognosis of disease ${ }^{4}$.

Recently the role of color Doppler ultrasonography in the diagnosis of ovarian malignancy has been a subject of discussion ${ }^{5}$. Conventionally, ovarian tumors are diagnosed on the basis of medical history, bimanual pelvic exam, trans-abdominal and transvaginal ultrasonography and CA 125 acts as an adjunct to diagnosis. The early diagnosis of cancer could also be made possible if neo-angiogenesis is regarded as a neoplastic marker for a tumour and by knowing the blood flow characteristics, one can predict whether the tumor is benign or malignant. Researchers have proposed Colour and pulsed Doppler flow imaging as methods that may be useful in differentiating benign from malignant ovarian masses ${ }^{6}$. Among the diagnostic modalities of ovarian tumors, Colour Doppler sonography (CDS) has ushered a new dimension. CDS is an ultrasound test that measures the blood flow characteristics of tumor blood vessels. Pelvic ultrasound scan, in conjunction with biochemical markers is recently being used to achieve greater accuracy in detecting ovarian malignancy. Transabdominal and Trans-vaginal sonography has no known biologic hazard and is a quicker method as well. It has been hypothesized that the detection of neo-vascularisation with abnormal, low-resistance blood flow peculiar to malignant tumors is possible, being characterized by angle-independent Doppler indices--PI and $\mathrm{RI}^{7}$. Salem et al. ${ }^{8,9}$ advocated colour and pulse Doppler sonography and studied tumour vascular characteristics by knowing their resistance index (RI) and pulsatility index (PI) value and they were found to be able to discriminate pre-operatively the benign and malignant tumors. Several researchers, Bourne et $\mathrm{al}^{10}$, Kurjak et $\mathrm{al}^{11}$, Weiner et al. ${ }^{1}$ and Fleischer et al. ${ }^{1}$ studied colour and pulse Doppler sonography and reported both high sensitivity and high specificity in distinguishing benign from malignant ovarian tumors.

Current study hypothesizes that Colour Doppler sonography is comparable to serum CA- 125 as a useful pre operative diagnostic tools to diagnose benign and malignant ovarian tumor. Hence, the aim of the research was to assess Colour Doppler Sonography and serum CA-125 level as diagnostic tool of ovarian tumour against a gold standard method (histopathological diagnosis) and also to compare between Colour Doppler Sonography and CA-125 as diagnostic tool of ovarian tumor. This study is aimed to assess and determine efficacy of different diagnostic tools to differentiate between benign and malignant ovarian tumour preoperatively. In the current study we have used colour Doppler and CA 125 and we have correlated these with postoperative histopathological findings.

\section{Materials and Methods}

This cross-sectional study was carried out at the Obstetrics and Gynecology department of BSMMU in collaboration with the Institute of Nuclear Medicine and Ultrasound (INMU), Pathology and Biochemistry Departments at BSMMU during the periods from July 2005 to July 2007. In the study, a total of 60 consecutive patients with ovarian tumor attending the Department of Obstetrics and Gynecology of BSMMU were recruited. Of the study participants $23.3 \%$ belong to $16-25$ year age group, $20 \%$ belong to $26-35$ years age group, $10 \%$ were of from 36-45 year age group and 23.30\% each were of 46-55 years and > 55 years age group. Mean age of the respondents were $42.13 \pm 17.6$ years. Age distribution suggested no specific age preference of ovarian tumor. All the patients recruited were from in-patient department and had undergone surgery. Following excision, routine histopathology revealed $43.30 \%$ malignant $(n=26)$ and $56.7 \%(\mathrm{n}=34)$ benign ovarian lesion.

Subjects were the patients diagnosed clinically as ovarian tumors and admitted in the hospital for operative treatment. The patients who were found to be unfit for major surgical operation, with inoperable ovarian tumors, patients with past history of major pelvic surgery for non-ovarian pathological fibrosis, vascular changes and patients who were not willing to participate were excluded from the study. The study proposal received approval of departmental ethical committee. The aims and objectives of the study along with its procedure, alternative diagnostic methods, risk and benefits of the studies were explained to the patients in easily understandable local language and then informed written consent were taken from each patient. All patients were assured of confidentiality and safety of the procedure by both the researchers and attending gynaecologist. Data were collected from primary source starting from the clinical history and bimanual pelvic examination, serum CA 125 levels, estimation of RI, PI, Novel Index by CDS and post-operative histopathological findings were then recorded. Sensitivity, specificity, accuracy, positive and negative predictive value of the diagnosis made by CDS, cutoff CA125, in the discrimination of the benign and malignant ovarian tumors were calculated considering histopathological diagnosis as gold standard according to the protocol described in Knapp and Miller ${ }^{13}$. 
Diagnostic procedures used:

Colour Doppler: Colour Doppler sonographic (CDS) technique is a non-invasive procedure, fast, reproducible and can be performed on a daily basis. This technique of CDS is highly specific as well as sensitive and thus it is used. In colour Doppler systems the pulses along each scan line are divided on return to the transducer, and some are used in providing image information and the rest are used to calculate the mean Doppler shift within small pixels of the image. This mean shift information is then coded on a colour scale and displayed as a colour map over the gray scale image. Flow towards the transducer is customarily coded red and the flow away, blue.

The resistance index $(R I)$ : The resistance index assesses arterial waveforms where there is no reverse flow component. The value is: $\mathrm{RI}=$ Peak systole-End diastole/Peak systole. And has the advantage that the value is independent of beam/vessel angle and only requires the measurement of two precisely defined points in the spectral display. The cut-off value for malignancy is $<0.5^{4,5,9,14}$. Pulsatility index (PI): This index was devised to determine quantitative energy in the oscillation of the waveform. The formula is: $\mathrm{PI}=$ Peak systole-End diastole/Mean peak value. It is particularly valuable in arteries in which there is diastolic flow reversal and can be calculated from a frequency trace without the need to know the beam/ vessels angles. Despite few limitations, the PI has gained wide clinical acceptance, particularly to determine the blood flow characteristics of abnormal blood vessels ${ }^{2}$. The cut-off value for malignancy is <1(5). CA 125: CA 125 is a tumor marker, a protein that is found in greater concentration in tumor cells than in other cells of the body. In particular, CA 125 is present in greater concentration in ovarian cancer cells than in other cells. CA 125 is usually measured from a blood sample. The cutoff value for CA 125 is less than 35 $\mathrm{U} / \mathrm{ml}$ for malignancy. Novel index: A novel index is proposed for the detection of ovarian malignancy combining resistance index (RI) obtained from newly formed vessels within the ovarian lesion and serum CA 125 level. The formula is: Novel Index = Resistance Index (R.I.) X100/Serum CA 125 level. Values below the cut-off value of 1.5 were associated with a high risk of ovarian malignancy. Novel index is found to have a good sensitivity and specificity for detection of ovarian malignancy ${ }^{16}$.

\section{Results}

Among the 60 study participants average age was $42.13 \pm 17.624$ years. And the size of the tumor of the patient ranged from $5.1 \mathrm{~cm}$ to $10 \mathrm{~cm}$. After surgical excision of the tumors, tissue histopathology was done and $43.3 \%$ (26) were found to be malignant $(n=26)$ and the rest were found to be benign. Histological type of tumor among the study subjects doesn't show any association with size of tumor.

Mucinous cystadenoma tops the benign tumor list with $47.1 \% \quad(n=16)$ followed by Benign serous cystadenoma (29.4\%). Among the malignant tumors majority (53.8\%) were Serous cystandenocarcinoma and Mucinous cyst adenocarcinoma $(23.1 \%)$. (Table I)

Using Receiver operative characteristics analysis the accuracy of Resistance index, Pulsatility Index, CA 125 and Novel Index in the diagnosis of ovarian tumor (Benign or malignant) were assessed (Table II). Area under the ROC curve demonstrates the good diagnostic accuracy of the indexes. The curve and area under the curve reveal that using the indexes is better than guessing. The area under the curve shows that the probability that the diagnosis made by the indexes for a randomly chosen positive case will exceed the result for a randomly chosen negative case. Cut-off used in the analysis closely matched the sensitivity and specificity (Table III \& Figure 1).

Table I: Histopathological type of tumor

\begin{tabular}{lccc}
\hline Benign & Frequency & $\%$ of & $\%$ of total \\
\hline Mucinous cystadenoma & 16 & $47.1 \%$ & $26.7 \%$ \\
Benign serous & 10 & $29.4 \%$ & $16.7 \%$ \\
Dermoid cyst & 06 & $17.6 \%$ & $10.0 \%$ \\
Brenners tumour & 02 & $05.9 \%$ & $03.3 \%$ \\
Total & 34 & $100 \%$ & \\
Malignant & & & \\
Serous & 14 & $53.8 \%$ & $23.3 \%$ \\
Mucinous cyst & 06 & $23.1 \%$ & $10.0 \%$ \\
Borderline serous & 02 & $07.7 \%$ & $03.3 \%$ \\
Malignant teratoma & 02 & $07.7 \%$ & $03.3 \%$ \\
Dysgerminoma & 02 & $07.7 \%$ & $03.3 \%$ \\
Total & 26 & $100 \%$ & \\
Grand total & 60 & & $100 \%$ \\
\hline
\end{tabular}

Table II Summery statistics of the indexes (Resistance Index, Pulsatility Index, CA 125, Novel Index.

\begin{tabular}{llcccc}
\hline Histopathology & Statistics & $\begin{array}{c}\text { Resistance } \\
\text { Index }\end{array}$ & $\begin{array}{c}\text { Pulsatility } \\
\text { Index }\end{array}$ & CA125 & $\begin{array}{c}\text { Novel } \\
\text { Index }\end{array}$ \\
\hline Benign & & 0.88 & 2.13 & 49.45 & 6.02 \\
& Mean & 0.43 & 1.26 & 74.14 & 7.43 \\
Malignant & SD & 0.47 & 1.14 & 263.10 & 0.64 \\
& Mean & 0.47 & & \\
& & & & & \\
Total & SD & 0.19 & 0.56 & 184.18 & 0.81 \\
& Mean & 0.70 & 1.70 & 142.03 & 3.69 \\
& SD & 0.40 & 1.13 & 169.85 & 6.20 \\
\hline
\end{tabular}


Figure 1: Receiver operative characteristics of resistance index, Pulsatility index CA 125 levels, Novel Index
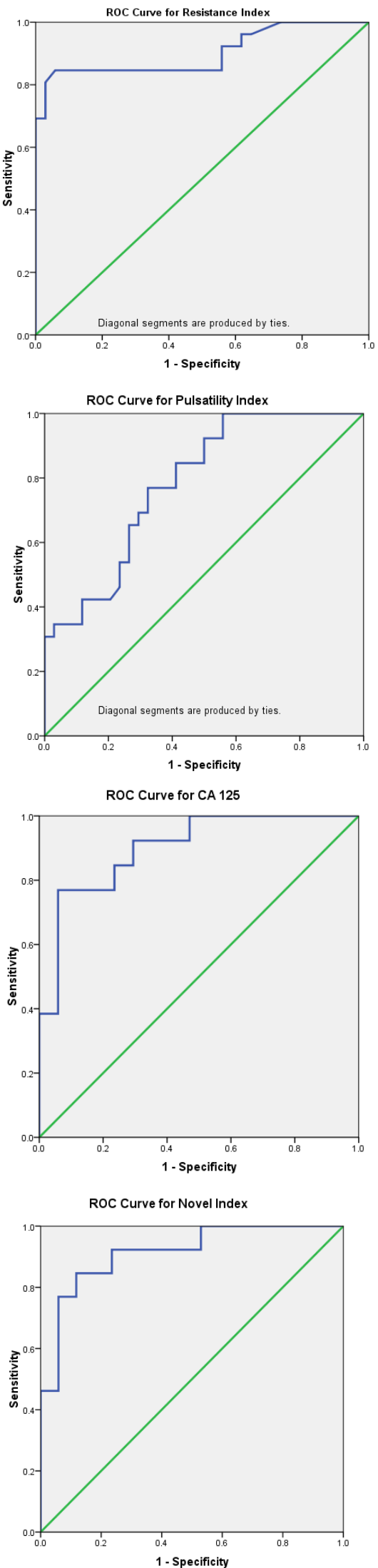

Table III: Area under the ROC Curve for Resistance index, Pulsatility index, CA 125 and Novel index

\begin{tabular}{lccccc}
\hline Variables & & \multicolumn{3}{c}{$95 \%$ CI } \\
& Area & SE & P value & Lower & Upper \\
\hline Resistance Index & 0.902 & 0.045 & 0.000 & 0.813 & 0.990 \\
Pulsatility Index & 0.783 & 0.058 & 0.000 & 0.670 & 0.896 \\
CA 125 & 0.900 & 0.039 & 0.000 & 0.824 & 0.977 \\
Novel Index & 0.914 & 0.037 & 0.000 & 0.842 & 0.986 \\
\hline
\end{tabular}

Under the nonparametric assumption

Null hypothesis: true area $=0.5$

\section{Gold standard: Histopathology}

With the Cut-off of <.5, Resistance index is found to be capable of detecting $92 \%$ of malignant cases (sensitivity 91.7), and could detect $89 \%$ (specificity 88.9)of benign cases correctly which translates in to $90 \%$ accuracy in the diagnosis of ovarian tumor. Predictive values for positive (84.6) and negative (94.1) tests were also found to be quite high. Pulsatility index was found to be moderate accuracy $(63.3 \%)$ with cutoff $<1$ for malignancy, however low predictive value for a positive test (38.5) questions its use. Both CA-125 and Novel Index showed similar level of sensitivity and specificity. Although Novel index is derivative of CA125, Novel index demonstrated better diagnostic accuracy and negative predictive value. Both the two tests with cut of respectively $>35$ and $<1.5$, were found to detect benign cases correctly in more than $90 \%$ occasions. Predictive value for positive value is high. Lower accuracy of CA 125 might have contributed by inappropriate cut-off determination. Following sensitivity analysis the cutoff for CA 125 was mandated as 83.58. With the value the sensitivity is $76.9 \%$ and the specificity is 94.1\% (Table IV).

Table IV: Cutoff used in the analysis

\begin{tabular}{lcccc}
\hline Statistics & $\begin{array}{c}\text { RI- } \\
\text { Index }\end{array}$ & $\begin{array}{c}\text { PI- } \\
\text { Index }\end{array}$ & $\begin{array}{c}\text { CA- } \\
125\end{array}$ & $\begin{array}{c}\text { Novel } \\
\text { Index }\end{array}$ \\
\hline Cut-off for malignant OT* & $<0.5$ & $<1$ & $>35$ & $<1.5$ \\
Sensitivity & 91.67 & 62.50 & 63.16 & 75.00 \\
Specificity & 88.89 & 63.64 & 90.91 & 92.86 \\
Accuracy & 90.00 & 63.33 & 73.33 & 83.33 \\
Positive predictive value & 84.62 & 38.46 & 92.31 & 92.31 \\
Negative predictive value & 94.12 & 82.35 & 58.82 & 76.47 \\
\hline *OT Ovarian tumor & & & &
\end{tabular}

*OT Ovarian tumor

\section{Discussion}

The symptoms of ovarian cancer are non-specific, as a result most patients tend to present in the later stages. Importantly prognosis is mostly dependent on stage at presentation ${ }^{17}$. Staging is surgical, but imaging, especially cross sectional imaging, has an important role in characterising adnexal masses as well as in assessing extent of disease prior to surgery. 
Among the two modalities used to diagnose ovarian tumor were Color Doppler sonography and CA 125 assay. There has been great interest about the role of ultrasonography in the diagnosis of ovarian malignancy, ${ }^{1,9,11-18}$. Color Doppler flow imaging provides non invasive method to evaluate blood flow patterns.

The cutoff used for resistance index (RI), Pulsatility index (PI), CA 125 level and Novel index are $<0.5,<1,<35$ and $<1.5$ respectively, for detecting ovarian malignancy. Using Receiver operative characteristics analysis the accuracy of Resistance index, Pulsatility Index, CA 125 and Novel Index in the diagnosis of ovarian tumor (Benign or malignant) were assessed. Area under the ROC curve demonstrates the good diagnostic accuracy of the indexes. The curve and area under the curve reveal that using the indexes is better than guessing. The area under the curve shows that the probability that the diagnosis made by the indexes for a randomly chosen positive case will exceed the result for a randomly chosen negative case. Cut-off used in the analysis closely matched the sensitivity and specificity.

ROC analysis also revealed cutoff similar to those used in the current study for resistance index (RI), Pulsatility index (PI), and Novel index for detecting ovarian malignancy. With the cut-off of <.5, Resistance index is found to be capable of detecting $92 \%$ of malignant cases, and could detect $89 \%$ of benign cases correctly which translates in to $90 \%$ accuracy in the diagnosis of ovarian tumor. Predictive values for positive and negative tests were also found to be quite high. Pulsatility index was also found to be moderate accuracy (63.3\%) with cutoff $<1$ for malignancy, however low predictive value for a positive test (38.5) questions its use.

Timor-Tritsch et al. ${ }^{19}$ have observed PI and RI values for benign and malignant ovarian tumors using CDS as 1.17 and 0.64 respectively for benign $\& 0.52$ and 0.39 respectively for malignant tumors.

A novel index proposed for the detection of ovarian malignancy combining resistance index (RI) obtained from newly formed vessels within the ovarian lesion and serum CA 125. Values below the cut-off value of 1.5 were associated with a high risk of ovarian malignancy. Both CA-125 and Novel Index showed similar level of sensitivity and specificity. Although Novel index is derivative of CA125, Novel index demonstrated better diagnostic accuracy and negative predictive value. Both the two tests with cut of respectively $>35$ and $<1.5$, were found to detect benign cases correctly in more than $90 \%$ occasions. Predictive value for positive value is high.
Our results suggested CDS as precise diagnostic tool with higher sensitivity and specificity for preoperative characterization and discrimination of benign from malignant tumors. Although CA-125 is a well reported and widely used tumor marker, we found this to be rather less sensitive of benign and malignant tumors. Its specificity is quite closer to null value and it makes mistake in detecting negative cases correctly in more than $40 \%$ occasions.

Lower accuracy of CA 125 might have contributed by inappropriate cut-off determination. Following sensitivity analysis the cutoff for CA 125 was mandated as 83.58 . With the value the sensitivity is $76.9 \%$ and the specificity is $94.1 \%$.

A similar study like ours reported cut-off values of 1.0 for PI and 0.4 or 0.7 for RI, with values below the cut-off suggestive of malignancy. They also found tumor marker CA 125 elevated in $80-85 \%$ of patients with epithelial ovarian cancer with levels over $35 \mathrm{U} / \mathrm{ml}$ suggestive of malignancy. In our study we used $0.35 \mathrm{U} / \mathrm{ml}$ as cutoff although the value was not found to be the best discriminator between benign and malignant ovarian cancer.

Kurjack et al. ${ }^{20}$ observed a significant correlation between CDS findings and histopathological findings. Regarding the PPV, it is equal in both study but the NPV is 7 percent lower in the current study. Kurjack et al. ${ }^{20}$ have shown that CDS offers 100 percent sensitivity and 99 percent specificity respectively in predicting benign and malignant ovarian tumours. The results obtained in the present study agree with the author that TV-CDS is highly specific for detecting ovarian cancer. Alcazar, Buckshee et $\mathrm{al}^{21}$. Buy et $\mathrm{al}^{22}$, and Fleischer et al. $^{23-25}$ concluded that CDS in pre-operative discrimination of benign and malignant ovarian masses is a very useful modality. CDS has been highly regarded as specific for the differentiation of adnexal masses. Our results strongly affiliate the notion.

Two methods, Color Doppler ultrasonography and CA125 excels in different tasks, RI is found to be more sensitive in detection of positive cases (Malignant) and CA125 is found to be more accurate in detection of negative cases (Benign). However a combination could be tried to make a better detection. Fleischer et al also proposed multiple methods to be performed together to improve the diagnostic accuracy. (Fleischer et al 1993).

Findings of this study recommends that multidisciplinary approach with different methods for early detection of ovarian malignancy, in context to the current study we recommend color 
Doppler sonography and ca125 to be used together for improving efficacy. Further study with larger sample can be done in population to establish the screening test standard for ovarian cancer.

\section{References}

1. Jemal, A., Murray, T., Ward, E., Samuels, A., Tiwari, R., Ghafoor, A., Feuer, E. L. \& Thun, M. J. (2005) $C A$ Cancer J. Clin. 55, 10-30.

2. Lu, K. H., Patterson, A. P., Wang. L, Marquez, R. T., Atkinson, E. N., Baggerly, K. A., Ramoth, L. R., Rosen, D. G., Liu, J., Hellstrom, I., et al. (2004) Clin. Cancer Res. 10, 3291-3300.

3. Weiner, Z., Beck, D., Sheitner, M., Borovik, R., BenShachar, M., Robinson, E., Brandes, J.,M. 1992, ‘ Screening for ovarian cancer in women with breast cancer with transvaginal sonography and color flow imaging'. J ultrasound med, vol.12, pp.387-393.

4. Barua, A., et al., Detection of Ovarian Tumors in Chicken by Sonography: A Step Toward Early Diagnosis in Humans? J Ultrasound Med, 2007. 26(7): p. $909-919$

5. Shy K and Dubinsky T: Is Color Doppler ultrasound useful in diagnosing ovarian cancer. Clin Obstetrics and Gynecology 1999; 42(4):902-15.

6. Antonić J, Rakar S. Validity of colour and pulsed Doppler US and tumour marker CA 125 in differentiation between benign and malignant ovarian masses. Eur J Gynaecol Oncol. 1996;17(1):29-35.

7. Hata, K; Hata, T; Intratumoral peak systolic velocity as a new possible predictor for detection of adnexal malignancy. American Journal of Obstetrics \& Gynecology. 172(5):1496-1500, May 1995.

8. Salem, S. The uterus \& adnexa. In Diagnostic Ultrasound. Rumack C.,M., Wilson, J.,R., Charboneau, J., W.(eds) 1998 Mosby, USA.

9. Salem, S., White, L.M., Lai, J. 1994 'Doppler sonography of adnexal masses: the predictive value of the pulsatility index in benign and malignant disease'. AJR, vol. 163, pp.1147-1150.

10. Bourne, T., Campbell, S., Steer, C., Whitehead, M., Collins, W.P., 1989 'Transvaginal colour flow imaging: a possible new screening technique for ovarian cancer', British medical journal, vol.299, pp.1367-70.

11. Kurjak, A., Kupesic S, Juckie, S., Schulman, L.,I., Socic, A., Zalud, I., Shalan, L., I.. 1992, 'Transvaginal ultrasound, color flow and Doppler waveform of the postmenopausal adnexal mass'. Obstet gynaecol. vol.1,pp.50-52.

12. Fleicher, A C Rogers WH, Rao BK, Kepple DM and Jones Hardware.(1991) Transvaginal color Doppler sonography of ovarian masses with pathological correlation. Ultrasound obstet. Gynecol, 1,275-8
13. Knapp, R., G., and Miller, M., C.,(eds) 1992, Clinical epidemiology and biostatistics.PP31-51.

14. Kurtz, A. B., Tsimikas, J. V., Tempany, C. M. C. , Hamper, U. M., Arger, P. H., Bree, R. L., Wechsler, et al. 1999, 'Diagnosis and Staging of Ovarian Cancer: Comparative Values of Doppler and Conventional US, $\mathrm{CT}$, and MR Imaging Correlated with Surgery and Histopathologic Analysis-Report of the Radiology Diagnostic Oncology Group'. Radiology. Vol.212, pp.19-27.

15. Meire, H.,B. Doppler in clinical ultrasound: Abdominal and general ultrasound. Cosgrove, D., Meire, H., Dewbury, K. (eds) 1993. Churchill livingstone, Edinburgh. PP67-65

16. Kupesic S, Vujisic S, Kurjak A, Mihaljevic D, Radosevic S. 2002, 'Preoperative assessment of ovarian tumors by CA 125 measurement and transvaginal color Doppler ultrasound'. Acta Med Croatica. vol.56,no.1,pp.3-10.

17. Pakkal, M.V. and M. Balogun, Imaging of ovarian cancer. Imaging, 2006. 18(1): p. 20-27.

18. Hata, K., Hata, T., Manabe, A., Kiato, M. 1992, 'Ovarian tumours of low malignant potential: Transvaginal Doppler ultrasound features. Gyne oncol, vol.45, pp.259-264

19. Timor-Trisch, I.E.,Lerner, J.P., Monteagudo, A., Santos, R. 1993, 'Transvaginal ultrasonographic characterization of ovarian masses by means of color flow- directed Doppler measurements and a morphologic scoring systems'. Am j obstet gynecol, vol. 168, pp.909-13.

20. Kurjak, A., Kupesic S, Juckie, S., Babic, D. 1997, 'A combined Doppler and morphological study of ovarian tumours'. Eur j obstet gynaeco reprod biol. vol.71,pp. $147-150$.

21. Alcazar, J.L., Errasti, T., Zornoza, A., Minguez, J.A., Galan, M.J.1996, Transvaginal color doppler ultrasonography and CA 125 in suspicious adnexal masses. Ultrasound obstet. Gynecol, vol.6, pp.1208-14.

22. Buy, J., N., Ghossain, M., A., Hugol, D., Hassen, K., Sciot, C., Truc, J-B., Poitout, P., Vadrot, D. 1996, 'Characterization of adnexal masses: combination of colour Doppler and conventional sonography compared with spectral doppler analysis alone and conventional sonography alone. AJR, vol.166, pp.385-393.

23. Fleischer, A.C., Culinan, J.A., Peery, C.V., Jones, H.W., 1996, 'Early detection of ovarian carcinoma with transvaginal color doppler ultrasonography'. Am J obstet gynecol, vol.174, pp. 101-6.

24. Fleischer, A.C., Rodgers, W.H., Kepple, D.M., Williams, L., Jones, H.W., Gross, P.R. 1992, 'Color doppler sonography of benign and malignant ovarian masses'. Radiographics', vol.12, pp.879-885.

25. Fleischer, A.C., Rodgers, W.H., Kepple, D.M., Williams, L., Jones, H.W. 1992, 'Color doppler sonography of ovarian masses: A multi parameter analysis.' . J Ultrasound Med', vol.12, pp.41-48. 